\title{
In vivo effect of imatinib on progression of cecal GIST-like tumors in exon 17-type c-kit knock-in mice
}

\author{
Takashi Ishikawa ${ }^{1,2}$, Norihiro Nakai ${ }^{1,3}$, Ning-Ning Liu ${ }^{1}$, Kazuhiro Shiba ${ }^{1,4}$, Koji Isozaki $^{1}$, Ikuo Matsuda ${ }^{1}$, Takashi Ito ${ }^{1}$, \\ Jiro Fujimoto ${ }^{3}$, Katsuyoshi Hatakeyama ${ }^{2}$, Tatsuo Kanda ${ }^{2}$ and Seiichi Hirota ${ }^{1}$
}

Two families with a germline Asp820Tyr mutation at exon 17 of the c-kit gene and multiple gastrointestinal stromal tumors (GISTs) have been reported. Recently, we generated a knock-in mouse model of the family, and mice with KIT-Asp818Tyr corresponding to human KIT-Asp820Tyr showed a cecal GIST-like tumor. In this report, we examined the in vivo effect of imatinib on tumor progression in knock-in mice. Imatinib of $100 \mu \mathrm{g} / \mathrm{g}$ body weight was administered to heterozygous (KIT-Asp818Tyr/ + ) mice orally for 7, 14 and 28 days, and cecal tumors were dissected. Both macroscopic size and the measured volume of cecal tumors were not significantly reduced after a 7-, 14- and 28-day administration of imatinib when compared with those before imatinib administration. Cell proliferation was assessed by Ki-67 immunohistochemistry and the labeling index significantly decreased after imatinib administration, but the value of the index after imatinib was only about half compared with that before imatinib. Western blotting and real-time PCR revealed that KIT expression was almost equivalent, but KIT phosphorylation was significantly but not completely inhibited in tumor tissues after 7, 14 and 28 days of imatinib administration when compared with that before imatinib administration. Phosphorylation of Akt and Stat 1 was accordingly inhibited after imatinib administration. Thus, imatinib seemed to inhibit in vivo tumor proliferation but not decrease tumor volume on this mouse model, probably because of an insufficient inhibition of phosphorylation of KIT and its downstream signaling molecules. These results suggested that progression of multiple GISTs in patients with germline Asp820Tyr might be partially controlled by imatinib and that model mice provide an opportunity to examine the effect of various other targeted drugs on in vivo tumor progression. Laboratory Investigation (2009) 89, 1161-1168; doi:10.1038/labinvest.2009.78; published online 27 July 2009

KEYWORDS: c-kit gene; exon 17; knock-in mouse; GIST-like tumor; imatinib

Gastrointestinal stromal tumors (GISTs) are the most common mesenchymal tumors of the human gut. Most GISTs express a receptor tyrosine kinase (TK), KIT, ${ }^{1,2}$ encoded by the proto-oncogene $c-k i t^{3-6}$ As interstitial cells of Cajal, which are possible pacemaker cells of the gastrointestinal movement, ${ }^{7}$ are also positive for KIT, ${ }^{8-10}$ GISTs are now considered to originate from interstitial cells of Cajal or from a precursor of them. KIT consists of an extracellular domain with five immunoglobulin-like repeats, a transmembrane domain, a juxtamembrane domain, and TK I and II domains split by the kinase insert. ${ }^{3-6}$ A ligand for KIT is stem cell factor. ${ }^{11-13}$ The binding of stem cell factor to KIT induces autophosphorylation of KIT, and its downstream pathways such as the Ras-MAPK, PI3K-Akt and Jak-Stat systems are activated to induce cell proliferation. The gain-of-function mutations of the c-kit gene cause a constitutive autophosphorylation of KIT and a subsequent activation of downstream signaling molecules without stem cell factor stimulation, and are considered to be associated with the pathogenesis of some tumor types. ${ }^{1,14,15}$ Most sporadic GISTs exhibit somatic gain-of-function mutations of the c-kit gene. ${ }^{1}$ The mutations are frequently found at exon 11 encoding the juxtamembrane domain, but the minority of them are found at exon 9, encoding the extracellular domain, at exon 13, encoding the TK I domain, or at exon 17, encoding the TK II domain. ${ }^{16-20}$

We first reported a family with multiple GISTs and a germline mutation of the c-kit gene at the juxtamembrane domain (deletion of one of two consecutive valine residues at codons 559 and 560 in exon 11, hereafter called KIT-del-Val559), and

\footnotetext{
${ }^{1}$ Department of Surgical Pathology, Hyogo College of Medicine, Nishinomiya, Japan; ${ }^{2}$ Division of Digestive and General Surgery, Niigata University Graduate School of Medical and Dental Sciences, Niigata, Japan; ${ }^{3}$ Department of Surgery, Hyogo College of Medicine, Nishinomiya, Japan and ${ }^{4}$ Department of Internal Medicine, Hyogo College of Medicine, Nishinomiya, Japan

Correspondence: Dr S Hirota, MD, PhD, Department of Surgical Pathology, Hyogo College of Medicine, 1-1 Mukogawa-cho, Nishinomiya, Hyogo 663-8501, Japan. E-mail: hiros@hyo-med.ac.jp

Received 12 January 2009; revised 10 June 2009; accepted 26 June 2009
} 
considered that the mutation might be the cause of familial and multiple GISTs. ${ }^{21}$ So far, at least 16 other families with multiple GISTs and specific germline mutations of the c-kit gene have been described. ${ }^{22-38}$ These families exhibited various types of mutations at exon $11,22,23,25,26,28-32,35,37$ one at exon 8 (KIT-del-Asp419), ${ }^{33}$ two at exon 13 (KITLys642Glu), ${ }^{24,36}$ and three at exon 17 (KIT-Asp820Tyr and KIT-Asn822Tyr). ${ }^{27,34,38}$ In 2003, Sommer $e t a l^{39}$ reported that they generated mice with a gain-of-function mutation of the c-kit gene at exon 11 (KIT-del-Val558) corresponding to human KIT-del-Val559 using a knock-in strategy, and heterozygotes exhibited a cecal GIST-like tumor. In 2005, Rubin et $a l^{40}$ generated another knock-in mouse strain with the c-kit gene exon 13-type mutation (KIT-Lys641Glu) corresponding to human KIT-Lys642Glu, and reported that heterozygotes and homozygotes exhibited a cecal GIST-like tumor. In 2008, we reported a mouse model of a human multiple GIST family with KIT-Asp820Tyr at exon 17 in the TK II domain of the c-kit gene that was generated using a knock-in strategy. ${ }^{41}$ In the model, heterozygous (KIT-Asp818Tyr/ + ) and homozygous (KIT-Asp818Tyr/KITAsp818Tyr) mice exhibited a cecal GIST-like tumor.

Imatinib is one of the small molecules of selective tyrosine kinase inhibitors that inhibits tyrosine kinase activity of KIT, platelet-derived growth factor receptors, and bcr-abl, ${ }^{42}$ and is now used widely for patients with GISTs and chronic myelogenous leukemia. ${ }^{43-45}$ It is well known that imatinib effectively inhibits the in vitro phosphorylation of various exon 11-type mutant KITs and the proliferation of cells harboring exon 11-type mutant KIT. ${ }^{46,47}$ In fact, most GISTs with various exon 11-type KIT mutations are well controlled by imatinib. ${ }^{48}$ Moreover, in 2006, Rossi et $a l^{49}$ demonstrated that imatinib showed a strong inhibitory effect on KIT signaling and cell proliferation index in the cecal GISTlike tumors of heterozygous exon 11-type knock-in mice (KIT-del-Val558/ + ). We previously reported that imatinib less effectively inhibited the phosphorylation of exon17-type mutant KIT (KIT-Asp818Tyr) than that of exon 11-type mutant KIT in vitro. ${ }^{47}$

In this report, we examined the in vivo inhibitory effect of imatinib on progression of the cecal GIST-like tumor of the exon 17-type knock-in mice. Although homozygotes have a larger tumor than do heterozygotes, most of them die within 10 weeks after birth. ${ }^{41}$ Therefore, we used heterozygotes in this examination. Imatinib did not show a reduction effect on tumor size and volume but demonstrated an inhibitory effect on phosphorylation of KIT signaling and on the cell proliferation index in vivo.

\section{MATERIALS AND METHODS}

\section{Mice and Drug Administration}

Twenty-five- to forty-eight-week-old female heterozygous (KITAsp818Tyr/ +) mice in a C57BL/6J background were used. Imatinib was kindly provided by Novartis (Basel, Switzerland), and was dissolved in distilled water at a concentration of
$5.0 \mathrm{mg} / \mathrm{ml}$. Rossi et $a l^{49}$ reported that they administered imatinib with $90 \mu \mathrm{g} / \mathrm{g}$ body weight per day to heterozygous exon 11-type (KIT-del-Val558/+) knock-in mice and the cecal tumors of the mice showed a remarkable decrease in cell proliferation index. Therefore, we orally administered imatinib of $50 \mu \mathrm{g} / \mathrm{g}$ body weight twice per day (total amount of $100 \mu \mathrm{g} / \mathrm{g}$ body weight per day) to our knock-in mice by an oral gavage. After 7 days, 2 weeks and 4 weeks, mice were killed $12 \mathrm{~h}$ after the last administration of the drug (7-, 14and 28-day groups). As a comparison, the heterozygous mice were dissected before imatinib treatment (0-day group). Four mice per group were used for histological examination, four for immunoblotting and three for real-time PCR.

\section{Histology, Immunohistochemistry, TUNEL Method and Approximate Tumor Volume Calculation}

Cecal tissues including the GIST-like tumors from heterozygous mice before and after imatinib administration were fixed with $10 \%$ formalin and embedded in paraffin. Sections (3- $\mu \mathrm{m}$ thick) were cut and used for conventional $\mathrm{H} \& \mathrm{E}$ staining and immunohistochemistry. Immunohistochemistry of Ki-67 was performed using a rat anti-mouse Ki-67 antigen monoclonal antibody (Ab) (M7249, DAKO, Glostrup, Denmark) and N-Histofine Simple Stain Mouse MAX PO (Rat) (Nichirei, Tokyo, Japan) with antigen retrieval. Ki-67 labeling index was calculated by the image analysis software, WinROOF (Mitani Corp., Fukui, Japan). Values of mean and standard error (s.e.) were calculated for each group, and Student's $t$-test was used to determine the significance of difference $(P<0.05)$ among the groups. Immunohistochemistry of cleaved caspase-3 was carried out using the cleaved caspase-3 (Asp175) Ab (9661, Cell Signaling Technology, Beverly, MA, USA) and ChemMate Envision Kit/HRP (DAB) System (DAKO). TUNEL method was performed by the DeadEnd Colorimetric TUNEL System (Promega Corporation, Madison, WI, USA), according to the manufacturer's instruction. Approximate tumor volume was calculated by measurement of tumor areas from multiple $\mathrm{H} \& \mathrm{E}$ sections. As the cecal tumors were linear and $1.5-2 \mathrm{~cm}$ in length, as shown in Figure 1, the tumors were crosscut at $2 \mathrm{~mm}$ intervals to 7-10 sections. The tumor area of each section $\left(A_{1}, A_{2}, A_{3} \ldots \ldots\right.$, and $\left.A_{n} m^{2}\right)$ was measured by WinROOF (Mitani Corp.), then the approximate tumor volume $\left(\mathrm{mm}^{3}\right)$ was calculated using the equation $2\left(\mathrm{~A}_{1}+\mathrm{A}_{2}\right) / 2+2\left(\mathrm{~A}_{2}+\mathrm{A}_{3}\right) /$ $2+\ldots \ldots+2\left(A_{n-2}+A_{n-1}\right) / 2+2\left(A_{n-1}+A_{n}\right) / 2=A_{1}+2 A_{2}+$ $2 \mathrm{~A}_{3}+\ldots \ldots+2 \mathrm{~A}_{\mathrm{n}-1}+\mathrm{A}_{\mathrm{n}}$. Values of mean and s.e. were calculated in each group, and Student's $t$-test was used to determine the significance of difference $(P<0.05)$ among the groups.

\section{Immunoblotting of KIT and its Possible Signaling Molecules, and Quantification of Signaling Density}

Only fresh GIST-like tumor tissues were carefully excised from the cecum of heterozygous mice for protein extraction. Lysis of the cecal tumor tissues was carried out with CelLytic-M lysis 

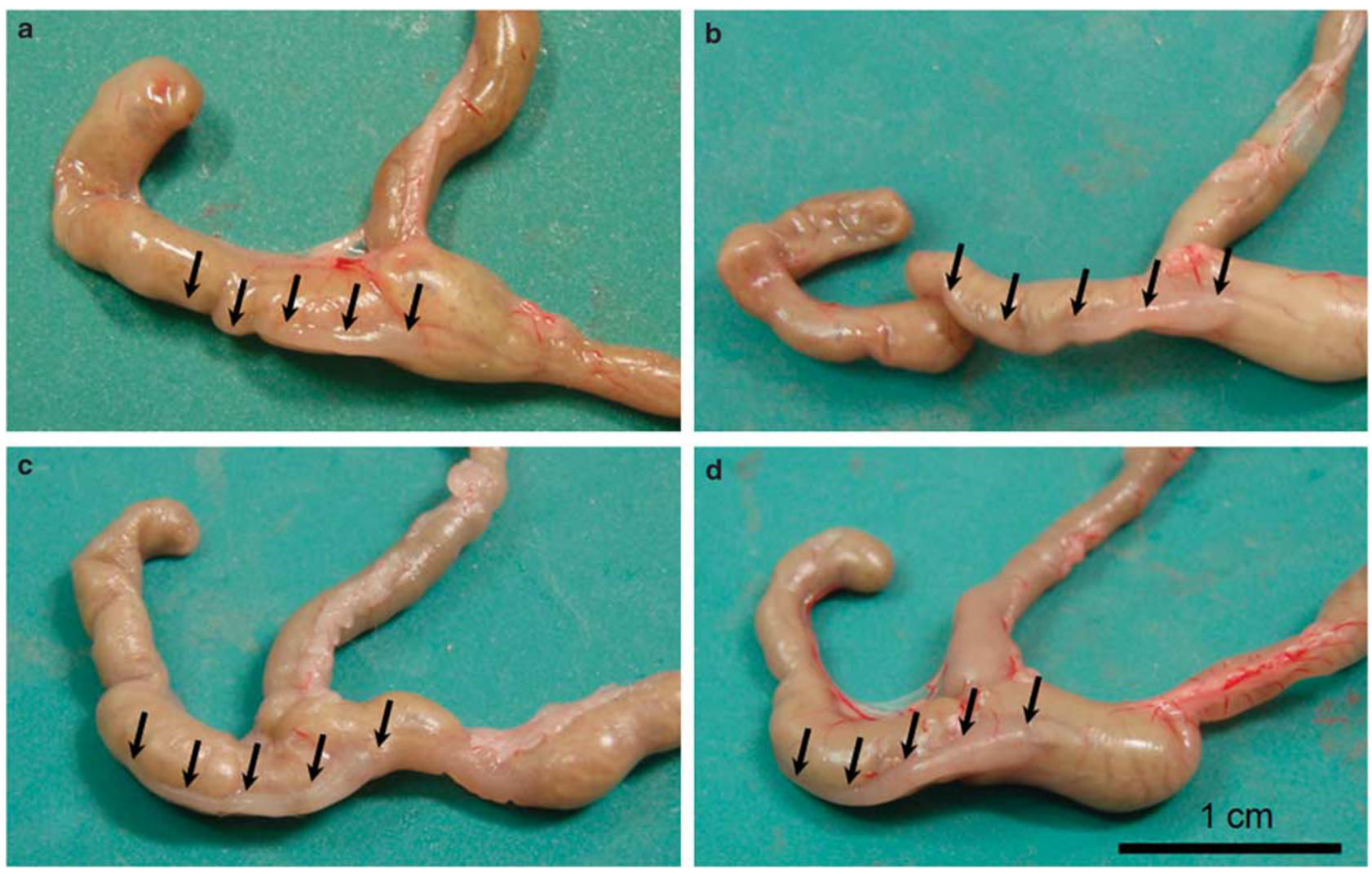

Figure 1 Representative macroscopic appearance of cecal tumors before and after imatinib administration. (a) Before administration of imatinib. (b) After a 1-week administration of imatinib. (c) After a 2-week administration of imatinib. (d) After a 4-week administration of imatinib. Macroscopic appearance including tumor size and color was not apparently changed in all the tumors. Arrows indicate cecal tumors.

buffer (Sigma-Aldrich, St Louis, MO, USA) with the addition of complete protease inhibitor cocktail tablets (Roche, Mannheim, Germany), phosphatase inhibitor cocktail (SigmaAldrich) and $1 \mu \mathrm{M} \mathrm{Na} \mathrm{Na}_{4}$. The extracted samples from four mice were mixed in each group for examination. SDSpolyacrylamide gel electrophoresis and immunoblotting were carried out as previously described. ${ }^{1}$ The Abs used were mouse anti-phospho-KIT Ab (pTyr823, Affinity BioReagents, Golden, CO, USA), rabbit anti-KIT polyclonal Ab (A4502; DAKO), phospho-Akt Ab (9271, Cell Signaling Technology), total Akt $\mathrm{Ab}$ (9272, Cell Signaling Technology), phospho-Stat1 Ab (9171, Cell Signaling Technology) and Stat1 Ab (9172, Cell Signaling Technology). The binding of Abs was detected by an ECL Western Blotting Detection System (Amersham Bioscience Corp.). The membranes used for the detection of phosphorylation of KIT, Akt and Statl were stripped and reprobed for the detection of total KIT, Akt and Stat1. Equivalent amounts of protein loading were shown by reprobing of actin (ab1801, Abcam, Cambridge, UK). We quantified the data of signaling blotting using an image analyzer (LAS-1000plus, FUJIFILM, Tokyo, Japan).

\section{RNA Extraction, cDNA Synthesis and Real-Time PCR}

Only fresh GIST-like tumor tissues were carefully excised from the cecum of heterozygous mice, and an RNeasy Mini
Kit (QIAGEN, Valencia, CA, USA) was used for total RNA extraction. Single-strand complementary DNA (cDNA) was synthesized using reverse transcriptase (Superscript II; GIBCO BRL, Grand Island, NY, USA) from total RNA, and real-time PCR using cDNA (20 ng) was carried out for amplification of murine c-kit cDNA by StepOne (Applied Biosystems) using SYBR Green. The primers used for real-time PCR were forward primer of $5^{\prime}$-CACAAAGATTTGCGATTTCG-3' (2437-2456) and reverse primer of $5^{\prime}$-CTTTCAAATGTGTACACGCA- $3^{\prime}$ (2552-2571). ${ }^{3}$ Data obtained from samples were used for the calculation of mean and s.e. values in each group. Mean and s.e. values of 7-, 14- and 28-day groups, and the s.e. value of the 0 -day group were expressed compared with the mean value of the 0 -day group as a control (1.0). Student's $t$-test was used to determine the significance of difference $(P<0.05)$ among groups.

\section{RESULTS \\ Macroscopic and Microscopic Change of Tumor and Measurement of Tumor Volume}

Macroscopically, the size of cecal tumor did not apparently change after a 7-, 14- and 28-day administration of imatinib when compared with that before imatinib administration (Figure 1a-d). Macroscopic changes other than tumor size were also not apparent. The approximate volumes of cecal 
tumor before imatinib administration (0 day) and those after the 7-, 14- and 28-day administration of imatinib were calculated by measurement of all tumor areas at $2 \mathrm{~mm}$ intervals according to the formula described in the Materials and Methods. As shown in Figure 2, difference in tumor volume was not statistically significant among the groups. Microscopic examination by H\&E staining did not show a notable change, such as necrosis, myxomatous change and hemorrhage, in all of the tumor tissues before imatinib

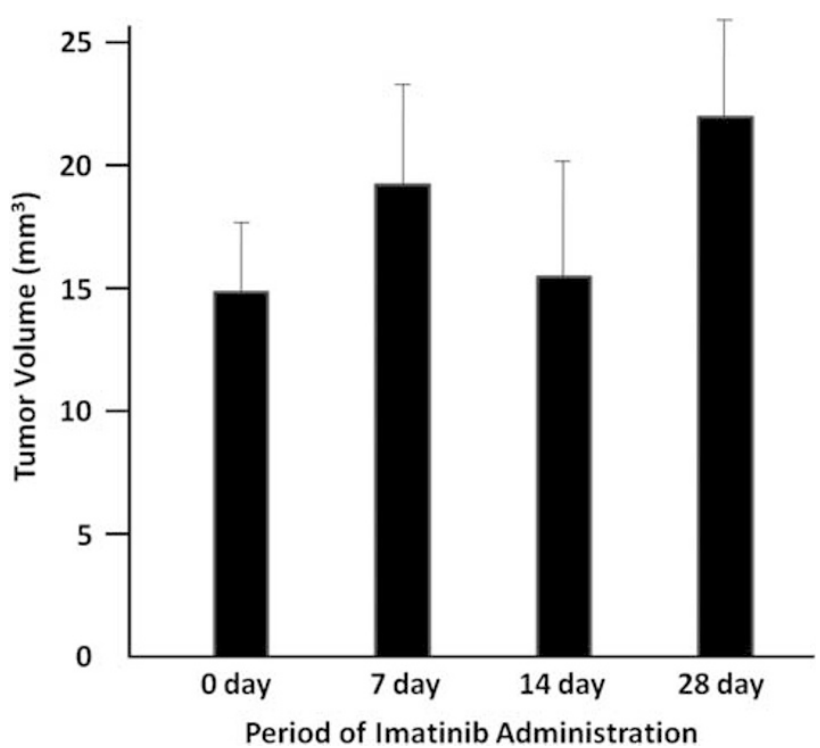

Figure 2 The calculated approximate tumor volume before administration of imatinib ( 0 day) and after administration of imatinib (7, 14 and 28 days). Difference in tumor volume was not statistically significant among the groups. Bars represent s.e. of the mean.
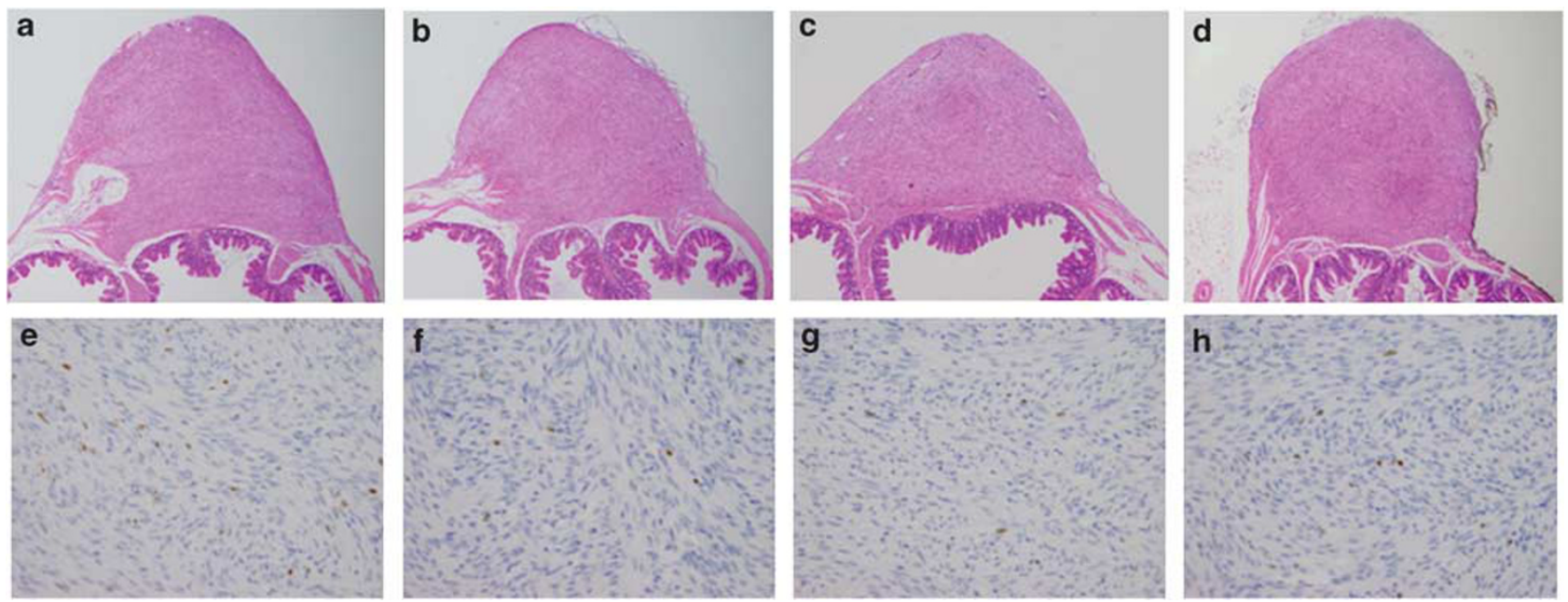

Figure 3 Representative microscopic appearance (a-d, H\&E staining) and Ki-67 immunohistochemistry (e-h) of cecal tumors before and after administration of imatinib. (a, e) Before administration of imatinib. (b, f) After a 1-week administration of imatinib. (c, $\mathbf{g}$ ) After a 2-week administration of imatinib. (d, h) After a 4-week administration of imatinib. Microscopic appearance was not apparently changed by H\&E staining. The number of Ki-67-positive cells was not high in all groups of tumors, but the number of them before imatinib administration (e) seemed to be more than that after imatinib administration (f-h). 


\section{KIT Signaling in Cecal Tumor after Imatinib Administration}

KIT expression and its phosphorylation were examined by western blotting in cecal tumors before and after 7, 14 and 28 days of imatinib administration. The expression of total

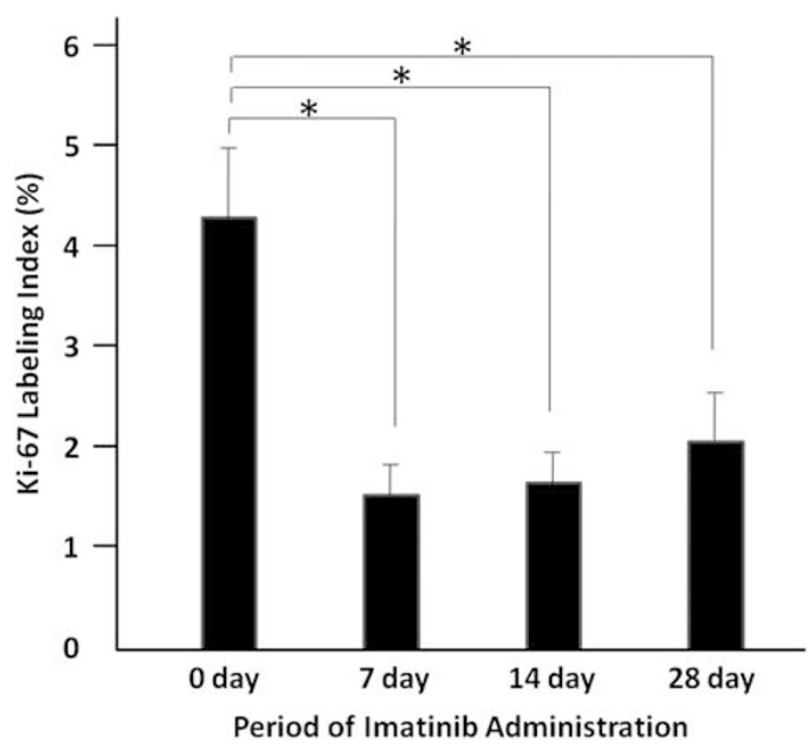

Figure 4 Comparison of the Ki-67 labeling index of cecal tumors before and after imatinib administration. Each Ki-67 labeling index counted in tumor tissues after 7-, 14- and 28-day imatinib administration was significantly smaller than that before imatinib administration ( 0 day) $\left({ }^{*} P<0.05\right)$. The difference of the Ki-67 labeling index among the 7-, 14- and 28-day groups was not statistically significant. Bars represent s.e. of the mean.
KIT was equivalent in the cecal tumors of all groups. The magnitude of phosphorylation of KIT after imatinib administration became weaker than that before imatinib administration (Figure $5 \mathrm{a}$ and $\mathrm{b}$ ).

The expression and phosphorylation of downstream KIT signaling molecules were also examined by western blotting in cecal tumors before and after imatinib administration. The equivalent expression of total Akt was observed in tumor tissues of all groups, and its phosphorylation after imatinib was also weaker than that before imatinib (Figure $5 \mathrm{c}$ and $d$ ). Similarly, the magnitude of expression of total Statl was similar among all groups of cecal tumors, and its phosphorylation after imatinib was weaker than that before imatinib, as observed in the expression and phosphorylation of KIT and Akt (Figure 5e and f). Equivalent amounts of protein loading were shown by blotting of actin (Figure $5 \mathrm{~g}$ ). The result of the signaling blot was quantified by image analyzer and data are shown in Figure $5 \mathrm{~h}$.

\section{Examination of c-kit mRNA Expression by Real-Time PCR} Expression of c-kit mRNA before and after 7-, 14- and 28-day administration of imatinib was examined by real-time PCR. The expression of c-kit mRNA before imatinib was not significantly altered compared with that after imatinib administration (Figure 6).

\section{DISCUSSION}

In this study using heterozygous knock-in mice with KITAsp818Tyr mutation, cecal tumor volume did not reduce but the Ki-67 labeling cell proliferation index in tumor tissues decreased significantly after 7-, 14- and 28-day imatinib
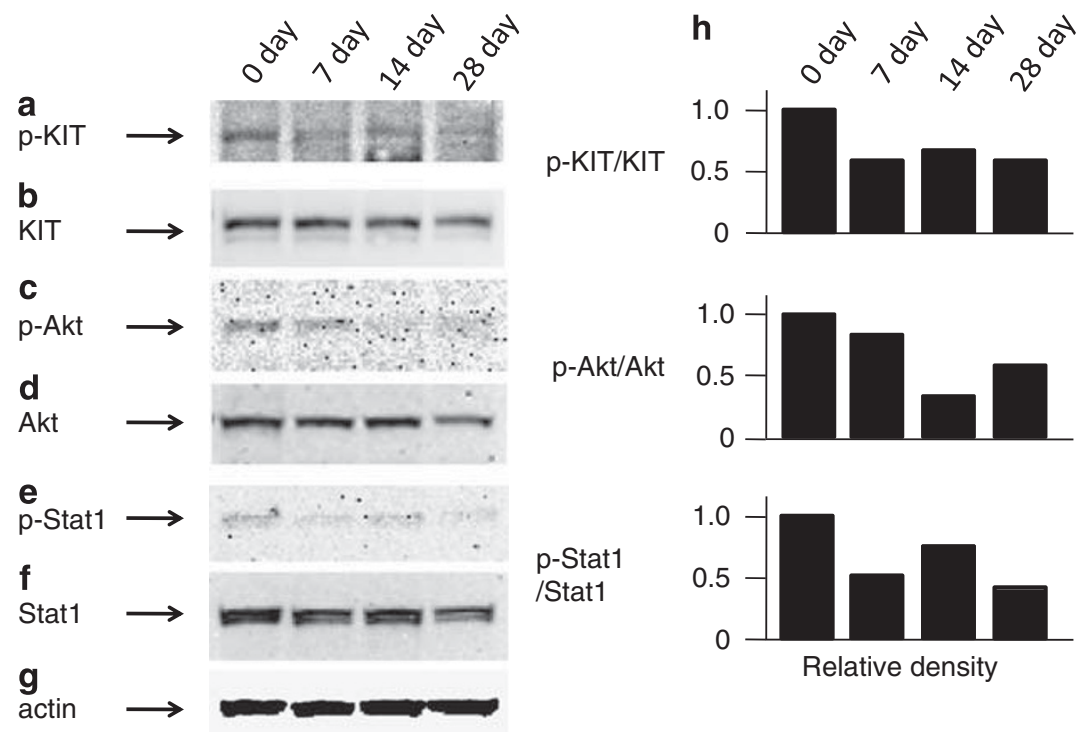

Figure 5 Expression and phosphorylation of KIT ( $\mathbf{a}$ and $\mathbf{b}$ ), Akt ( $\mathbf{c}$ and $\mathbf{d}$ ) and Stat1 (e and $\mathbf{f}$ ) in cecal tumors of heterozygotes before and after imatinib administration. Phosphorylation of KIT (a) after imatinib administration (7, 14 and 28 days) was weaker than that before imatinib administration ( 0 day), although expression of total KIT (b) was equivalent among the groups. Similarly, the phosphorylation of Akt (c) and Stat 1 (e) after imatinib administration $(7,14$ and 28 days) was weaker than that before imatinib administration ( 0 day), although the expression of total Akt (d) and Stat1 (f) was similar among the groups. Equivalent loading of protein was shown by blotting of actin (g). Result of the signaling blot was quantified by image analyzer, and the relative density of phosphorylated protein/total protein was demonstrated, as each datum of day 0 was 1.0 (h). 


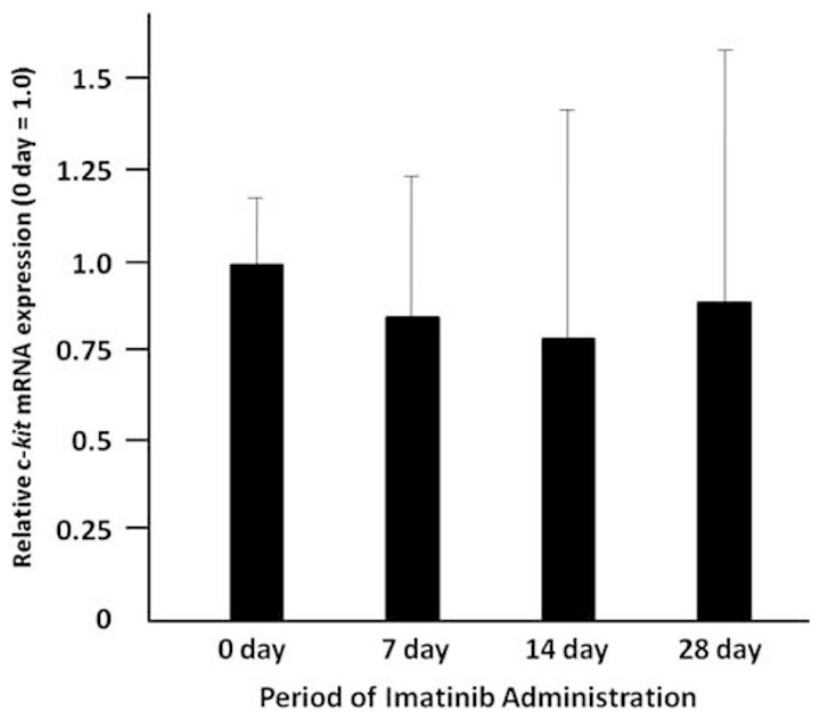

Figure 6 Expression of c-kit mRNA before and after imatinib administration examined by real-time PCR. The mean value of day 0 was used as control (1.0). Expression of c-kit mRNA after imatinib (7, 14 and 28 days) was not significantly altered compared with that before imatinib administration (0 day). Bars represent s.e. of the mean.

administration when compared with those before imatinib administration. As human KIT-Asp820Tyr found in familial GISTs corresponds to mouse KIT-Asp818Tyr, imatinib might be effective for patients with multiple GISTs on stabilization of tumor progression.

Imatinib was administered with $100 \mu \mathrm{g} / \mathrm{g}$ body weight per day for 7, 14 and 28 days in this study. There is a possibility that the duration of drug treatment was not sufficient for tumor volume reduction in exon 17-type knock-in mice. A much longer administration to knock-in mice might reveal the long-term effect of imatinib on reduction of tumor volume. Similarly, there is a possibility that the amount of drug was not sufficient for tumor volume reduction in knock-in mice. A more administration of the drug to knockin mice might reveal the high-dose effect of imatinib on reduction of tumor volume.

We showed that imatinib partially inhibited KIT phosphorylation in cecal tumors of heterozygous exon 17-type (KIT-Asp818Tyr) knock-in mice in this study. In 2006, Rossi et $a l^{49}$ reported that imatinib rapidly and almost completely inhibited KIT phosphorylation in cecal tumors of heterozygous exon 11-type (KIT-del-Val558/+) knock-in mice. Previously we showed that imatinib almost completely inhibited the phosphorylation of KIT in cells expressing exon 11-type mutant KIT (KIT-del-Val558\&Val559), but partially inhibited it in cells expressing exon 17-type mutant KIT (KIT-Asp818Tyr) at a concentration of $1 \mu \mathrm{M}$ in vitro. ${ }^{47}$ The in vivo results obtained by Rossi $e t a l^{49}$ and by this study were consistent with our previous in vitro data. ${ }^{47}$

In this study, imatinib partially inhibited cell proliferation in cecal tumors of heterozygous exon 17-type (KIT-Asp818Tyr/ + ) knock-in mice. On the other hand, it has been reported by Rossi et $a l^{49}$ that imatinib almost completely inhibited cell proliferation in cecal tumors of heterozygous exon 11-type (KIT-del-Val558/+) knock-in mice. These results were parallel to the inhibitory effect of imatinib on KIT phosphorylation in vivo. Our in vitro examination showed that the degree of inhibition of KIT phosphorylation was correlated with the degree of inhibition of tumor cell proliferation. ${ }^{47}$ The present result and the previous result by Rossi et a $l^{49}$ indicated that the degree of inhibition of in vivo KIT phosphorylation was also closely related to the degree of inhibition of in vivo tumor cell proliferation.

We could not detect an apparent histological response such as necrosis, fibrosis and myxoid change in the cecal tumors of heterozygous exon 17-type (KIT-Asp818Tyr/+) knock-in mice even after 28 days of imatinib administration. In the cecal tumors of heterozygous exon 11-type (KIT-del-Val558/+) knock-in mice, some histological changes have been reported after 7 days of imatinib treatment. ${ }^{49}$ A stronger inhibitory effect of imatinib on KIT phosphorylation and cell proliferation in exon 11-type knock-in mice than in exon 17-type knock-in mice might contribute to the results. In fact, increased apoptosis with increased caspase 3-positive cells in cecal tumors has been demonstrated in exon 11-type knock-in mice. ${ }^{49}$ In this study, on the other hand, we showed that apoptotic change was not apparent even in post-imatinib tumors. There is a possibility that imatinib partially inhibited tumor cell proliferation, but the effect was not enough to induce apoptosis.

In this study, real-time PCR of c-kit cDNA and western blotting of the total KIT protein revealed that the level of KIT expression was similar in tumor tissues both before and after imatinib treatment. Imatinib essentially did not seem to affect both c-kit gene transcription and KIT translation. The KIT expression might decrease if stronger histological responses of tumors, such as degeneration and necrosis, were observed.

We showed that phosphorylation of downstream signaling molecules of Akt and Statl was partially inhibited by imatinib treatment as observed in KIT phosphorylation. Rossi et $a l^{49}$ reported that imatinib strongly inhibited the phosphorylation of Akt and other downstream signaling molecules. Akt is a common downstream signaling molecule affected by imatinib treatment in both exon 11-type and exon 17-type knock-in mice.

Imatinib is an extremely effective agent in the treatment of GIST patients with exon 11-type c-kit gene mutation. ${ }^{48}$ As indicated in this report, however, the in vivo inhibitory effect of imatinib on exon 17-type mutant KIT was not so strong. Moreover, secondary imatinib-resistant clones often develop in GIST patients during long-term imatinib treatment through secondary mutations of the c-kit gene at exon 13, 17 or $18 .^{50-52}$ Many targeted drugs for various mutations, including secondary mutations, are being developed. The exon 17-type (KIT-Asp818Tyr) c-kit gene knock-in mice 
are useful models to assess the in vivo effect and safety of such various targeted drugs.

\section{ACKNOWLEDGEMENTS}

We thank Ms Y Hashikura and Ms M Ohkouchi for their technical assistance. This work was supported in part by grants from the Ministry of Education, Culture, Sports, Science and Technology of Japan (17013082, 19590410, and 19590411).

\section{DISCLOSURE/CONFLICT OF INTEREST}

The authors declare no conflict of interest.

1. Hirota S, Isozaki K, Moriyama Y, et al Gain-of-function mutations of c-kit in human gastrointestinal stromal tumors. Science 1998;279:577-580.

2. Kindblom LG, Remotti $H E$, Aldenborg $F$, et al Gastrointestinal pacemaker cell tumor (GIPACT): gastrointestinal stromal tumors show phenotypic characteristics of the interstitial cells of Cajal. Am J Pathol 1998;152:1259-1269.

3. Yarden $Y$, Kuang WJ, Yang-Feng $T$, et al Human proto-oncogene c-kit: a new cell surface receptor tyrosine kinase for an unidentified ligand. EMBO J 1987;6:3341-3351.

4. Chabot B, Stephenson DA, Chapman VM, et al The proto-oncogene c-kit encoding a transmembrane tyrosine kinase receptor maps to the mouse W locus. Nature 1988;335:88-89.

5. Qiu FH, Ray HP, Brown Y, et al Primary structure of c-kit: Relationship with the CSF-1/PDGF receptor kinase family-oncogenic activation of $\mathrm{v}$-kit involves deletion of extracellular domain and C terminus. EMBO J 1988;7:1003-1111.

6. Geissler EN, Ryan MA, Housman DE. The dominant-white spotting (W) locus of the mouse encodes the c-kit proto-oncogene. Cell 1988;55:185-192.

7. Thomsen L, Robinson TL, Lee JC, et al Interstitial cells of Cajal generate a rhythmic pacemaker current. Nat Med 1998;4:848-851.

8. Maeda H, Yamagata A, Nishikawa S, et al Requirement of c-kit for development of intestinal pacemaker system. Development 1992;116:369-375.

9. Huizinga JD, Thuneberg $L$, Kluppel $M$, et al $W / k i t$ gene required for interstitial cells of Cajal and for intestinal pacemaker activity. Nature 1995;373:347-349.

10. Isozaki K, Hirota S, Nakama A, et al Disturbed intestinal movement, bile reflux to the stomach, and deficiency of c-kit-expressing cells in Ws/Ws mutant rats. Gastroenterology 1995;109:456-464.

11. Williams DE, Eisenman J, Baird $A$, et al Identification of a ligand for the c-kit proto-oncogene. Cell 1990;63:167-174.

12. Flanagan JG, Leder $P$. The kit ligand: a cell surface molecule altered in steel mutant fibroblasts. Cell 1990;63:185-194.

13. Zsebo KM, Williams DA, Geissler EN, et al Stem cell factor is encoded at the $\mathrm{SI}$ locus of the mouse and is the ligand for the c-kit tyrosine kinase receptor. Cell 1990;63:213-224.

14. Furitsu $T$, Tsujimura $T$, Tono $T$, et al Identification of mutations in the coding sequence of the proto-oncogene c-kit in a human mast cell leukemia cell line causing ligand-independent activation of c-kit product. J Clin Invest 1993;92:1736-1744.

15. Tian Q, Frierson Jr HF, Krystal GW, et al Activating c-kit gene mutations in human germ cell tumors. Am J Pathol 1999;154:1643-1647.

16. Lux ML, Rubin BP, Biase $T L$, et al KIT extracellular and kinase domain mutations in gastrointestinal stromal tumors. Am J Pathol 2000;156:791-795.

17. Lasota J, Wozniak A, Sarlomo-Rikala M, et al Mutations in exons 9 and 13 of KIT gene are rare events in gastrointestinal stromal tumors. Am J Pathol 2000;157:1091-1095.

18. Hirota S, Nishida T, Isozaki K, et al Gain-of-function mutation at the extracellular domain of KIT in gastrointestinal stromal tumours. J Pathol 2001;193:505-510.

19. Kinoshita K, Isozaki K, Hirota S, et al c-kit gene mutation at exon 17 or 13 is very rare in sporadic gastrointestinal stromal tumors. J Gastroenterol Hepatol 2003;18:147-151.

20. Kinoshita $K$, Hirota $S$, Isozaki $K$, et al Characterization of tyrosine kinase I domain c-kit gene mutation Asn655Lys newly found in primary jejunal gastrointestinal stromal tumor. Am J Gastroenterol 2007;102: 1134-1136.

21. Nishida $T$, Hirota $S$, Taniguchi $M$, et al Familial gastrointestinal stromal tumours with germline mutation of the KIT gene. Nature Genet 1998;19:323-324.

22. O'Brien $\mathrm{P}$, Kapusta L, Dardick I, et al Multiple familial gastrointestinal autonomic nerve tumors and small intestinal neuronal dysplasia. Am J Surg Pathol 1999;23:198-204.

23. Hirota $S$, Okazaki T, Kitamura $Y$, et al Cause of familial and multiple gastrointestinal autonomic nerve tumors with hyperplasia of interstitial cells of Cajal is germline mutation of the c-kit gene. Am J Surg Pathol 2000;24:326-327.

24. Isozaki K, Terris B, Belghiti J, et al Germline-activating mutation in the kinase domain of KIT gene in familial gastrointestinal stromal tumors. Am J Pathol 2000;157:1581-1585.

25. Maeyama $\mathrm{H}$, Hidaka $\mathrm{E}$, Ota $\mathrm{H}$, et al Familial gastrointestinal stromal tumor with hyperpigmentation: association with a germline mutation of the c-kit gene. Gastroenterology 2001;120:210-215.

26. Beghini A, Tibiletti MG, Roversi $G$, et al Germline mutation in the juxtamembrane domain of the kit gene in a family with gastrointestinal stromal tumors and urticaria pigmentosa. Cancer 2001;92:657-662.

27. Hirota $\mathrm{S}$, Nishida $\mathrm{T}$, Isozaki $\mathrm{K}$, et al Familial gastrointestinal stromal tumors associated with dysphagia and novel type germline mutation of KIT gene. Gastroenterology 2002;122:1493-1499.

28. Robson ME, Glogowski E, Sommer G, et al Pleomorphic characteristics of a germ-line KIT mutation in a large kindred with gastrointestinal stromal tumors, hyperpigmentation, and dysphagia. Clin Cancer Pathol 2004;10:1250-1254

29. Carballo M, Aguilar F, Pol MA, et al Novel c-KIT germline mutation in a family with gastrointestinal stromal tumors and cutaneous hyperpigmentation. Am J Med Genet A 2005;132:361-364.

30. Li FP, Fletcher JA, Heinrich MC, et al Familial gastrointestinal stromal tumor syndrome: phenotype and molecular features in a kindred. J Clin Oncol 2005:23:2735-2743.

31. Kim HJ, Lim SJ, Park K, et al Multipe gastrointestinal stromal tumors with a germline c-kit mutation. Pathol Int 2005;55:655-659.

32. Tarn C, Merkel E, Canutescu AA, et al Analysis of KIT mutations in sporadic and familial gastrointestinal stromal tumors: therapeutic implications through protein modeling. Clin Cancer Res 2005;11:2005.

33. Hartmann K, Wardelmann E, Ma Y, et al Novel germline mutation of KIT associated with familial gastrointestinal stromal tumors and mastocytosis. Gastroenterology 2005;129:1042-1046.

34. O'Riain C, Corless CL, Heinrich MC, et al Gastrointestinal stromal tumors: insights from a new familial GIST kindred with unusual genetic and pathologic features. Am J Surg Pathol 2005;29:1680-1683.

35. Kang DY, Park CK, Choi JS, et al Multiple gastrointestinal stromal tumors: clinicopathologic and genetic analysis of 12 patients. Am J Surg Pathol 2007;31:224-232.

36. Graham J, Debiec-Rychter M, Corless $\mathrm{CL}$, et al Imatinib in the management of multiple gastrointestinal stromal tumors associated with a germline KIT K642E mutation. Arcch Pathol Lab Med 2007;131:1393-1396.

37. Wozniak A, Rutkowski $P$, Sciot $R$, et al Rectal gastrointestinal stromal tumors associated with a novel germline KIT mutation. Int J Cancer 2008;122:2160-2164.

38. Thalheimer $A$, Schlemmer $M$, Bueter $M$, et al Familial gastrointestinal stromal tumors caused by the novel KIT exon 17 germline mutation N822Y. Am J Surg Pathol 2008;32:1560-1565.

39. Sommer G, Agosti V, Ehlers I, et al Gastrointestinal stromal tumors in a mouse model by target mutation of the Kit receptor tyrosine kinase. Proc Natl Acad Sci USA 2003;100:6706-6711.

40. Rubin BP, Antonescu C, Scott-Browne JP, et al A knock-in mouse model of gastrointestinal stromal tumor harboring KIT K641E. Cancer Res 2005;65:6631-6639.

41. Nakai N, Ishikawa T, Nishitani A, et al A mouse model of a human multiple GIST family with KIT-Asp820Tyr mutation generated by a knock-in strategy. J Pathol 2008;214:302-311.

42. Buchdunger $\mathrm{E}$, Cioffi $\mathrm{CL}$, Law $\mathrm{N}$, et al Abl protein-tyrosine kinase inhibitor STI571 inhibits in vitro signal transduction mediated by c-KIT and platelet-derived growth factor receptors. J Pharmaco Exp Ther 2000;295:139-145. 
43. Druker BJ, Talpaz M, Resta DJ, et al Efficacy and safety of a specific inhibitor of the BCR-ABL tyrosine kinase in chronic myeloid leukemia. N Engl J Med 2001;344:1031-1037.

44. Van Oosterom AT, Judson I, Verweiji J, et al Safety and efficacy of imatinib (STI571) in metastatic gastrointestinal stromal tumours: a phase I study. Lancet 2001;358:1421-1423.

45. Demetri GD, von Mehren $M$, Blanke CD, et al Efficacy and safety of imatinib mesylate in advanced gastrointestinal stromal tumors. N Engl J Med 2002;347:472-480.

46. Heinrich MC, Griffith DJ, Druker BJ, et al Inhibition of c-KIT receptor tyrosine activity by STI571, a selective tyrosine kinase inhibitor. Blood 2000;96:925-932.

47. Chen $\mathrm{H}$, Isozaki $\mathrm{K}$, Kinoshita $\mathrm{K}$, et al Imatinib inhibits various types of activating mutant KIT found in gastrointestinal stromal tumors. Int J Cancer 2003;105:130-135.

48. Heinrich MC, Owzar K, Corless CL, et al Correlation of kinase genotype and clinical outcome in the North American Intergroup Phase III
Trial of imatinib mesylate for treatment of advanced gastrointestinal stromal tumor: CALGB 150105 Study by Cancer and Leukemia Group B and Southwest Oncology Group. J Clin Oncol 2008;26: 5360-5367.

49. Rossi F, Ehlers I, Agosti V, et al Oncogenic Kit signaling and therapeutic intervention in a mouse model of gastrointestinal stromal tumor. Proc Natl Acad Sci USA 2006;103:12843-12848.

50. Antonescu CR, Besmer $P$, Guo $T$, et al Acquired resistance to imatinib in gastrointestinal stromal tumor occurs through secondary gene mutation. Clin Cancer Res 2005;11:4182-4190.

51. Heinrich MC, Corless CL, Blanke CD, et al Molecular correlates of imatinib resistance in gastrointestinal stromal tumors. J Clin Oncol 2006;24:4764-4774.

52. Nishida T, Kanda T, Nishitani A, et al Secondary mutations in the kinase domain of the KIT gene are predominant in imatinibresistant gastrointestinal stromal tumor. Cancer Sci 2008;99: 799-804. 